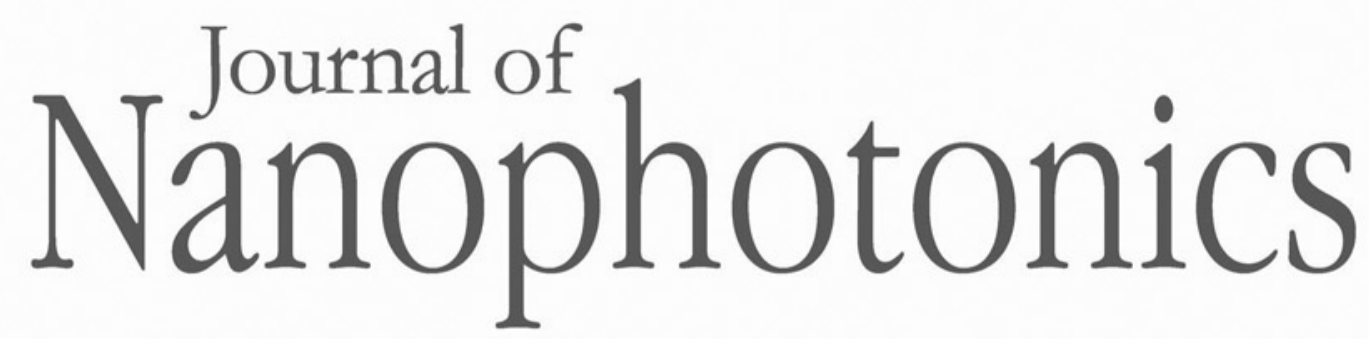

Nanophotonics.SPIEDigitalLibrary.org

\title{
Special Section Guest Editorial: Semiconductor UV Photonics
}

\author{
Tien Khee Ng \\ Jianchang Yan
}




\title{
Special Section Guest Editorial: Semiconductor UV Photonics
}

\author{
Tien Khee $\mathrm{Ng}^{\mathrm{a}}$ and Jianchang $\mathbf{Y a n}^{\mathrm{b}, \mathrm{c}, \mathrm{d}, \mathrm{e}}$ \\ ${ }^{a}$ King Abdullah University of Science and Technology (KAUST), Photonics Laboratory, \\ Thuwal, Saudi Arabia \\ ${ }^{\mathrm{b}}$ Chinese Academy of Sciences, Institute of Semiconductors, Research and Development Center \\ for Solid State Lighting, Beijing, China \\ ${ }^{c}$ University of Chinese Academy of Sciences, Beijing, China \\ ${ }^{\mathrm{d} B e i j i n g ~ E n g i n e e r i n g ~ R e s e a r c h ~ C e n t e r ~ f o r ~ t h e ~ T h i r d-G e n e r a t i o n ~ S e m i c o n d u c t o r ~ M a t e r i a l s ~ a n d ~}$ \\ Application, Beijing, China \\ ${ }^{\text {e}}$ State Key Laboratory of Solid State Lighting, Beijing, China
}

The AlGaN-based ultra-wide bandgap semiconductor has entered the realm of relative maturity as evident in the foundry production of ultraviolet range light-emitting diodes (LEDs). These LEDs offer the significant advantage of having compact, small footprint features for a wide range of applications requiring the utility of ultraviolet emission spectrum. While this is encouraging, a multitude of scientific and technological challenges remain. The demonstration of devices operating in the full ultraviolet-spectrum necessitates the meticulous and painstaking epitaxy and process development in circumventing issues that are essentially related to crystal symmetry, polarization field, dopant activation efficiency, lattice-mismatch, epitaxy cracking, quantum efficiencies, and substrate considerations. Other classes of AlInGaN-based and ZnO-based photonic devices are equally demanding in the development of photonic elements, semiconductors and device physics. Various aspects of the above discussion were, to a large extent, covered by the collection of articles in the present special section. Hence, readers will benefit from the extensive results, knowledge, and the best practices in the existing technological advances, as reported in the collection of papers. Moreover, readers are urged to challenge the status-quo of the current methods and knowhow reported in the review articles, in their endeavors on fabricating photonic devices operating in the ultraviolet regime. Practical and innovative solutions that simplify the methods of fabrication and resolve the persistent challenges in ultraviolet-spectrum devices, as mentioned above, are still required. This special section hopes to further elicit thoughts from the semiconductor, optoelectronics, and photonics community for crystallizing creative solutions in making better devices.

The Journal of Nanophotonics Special Section on Semiconductor UV Photonics comprises three review articles and nine contributed articles. These articles offer readers both the breadth and depth of the latest advances in the design, growth, fabrication, and implementation of optically pumped or electrically injected devices and structures made of ultra-wide bandgap and wide bandgap nitride- and oxide-based semiconductor materials.

The review articles are: (1) a critical review, by Yanan Guo et al., on the current practices in enhancing light-extraction, especially that of TM-polarized light, in AlGaN-based LEDs grown by metal-organic chemical vapor deposition (MOCVD); (2) a survey article by Mohd Sharizal Alias et al. on the state-of-the-art nanophotonics considerations and fabrication approaches in addressing various challenges in ultraviolet-emitting photonic devices, optical elements and nanostructures; and (3) a perspective review article by Jung-Wook Min et al. on the critical considerations and innovative characterization techniques for unleashing the potential of molecular beam epitaxy (MBE) grown AlGaN-based ultraviolet-spectrum self-assembled nanowires devices, including LEDs and photodetectors.

The following further introduces the contributed articles, which covers the AlGaN-based epitaxy growth on both conventional and non-conventional substrates (silicon), simulation of AlN/GaN heterointerface point-defects, nanophotonic-structure implementation and simulation (photonic crystals, meta-lens, nano-porous structure, substrate-free microcavity, whisperinggallery modes microdisk), and characterization of physical phenomenon, such as the magnetoexciton recombination, in $\mathrm{ZnO}$. 
Zhongming Zheng et al. fabricated a substrate-free microcavity device based on AlGaNquantum-dots stacks, sandwiched between the top and bottom $\mathrm{HfO}_{2} / \mathrm{SiO}_{2}$ distributed Bragg reflectors (DBRs). The investigation sheds light on the optical scattering losses in vertical cavity surface emitting laser (VCSEL) emitting in the ultraviolet-B/C wavelength regime. In the attempt to extend the whispering-gallery modes for ultraviolet-C emission spectrum, Yiyun Zhang et al. implemented AlN/AlGaN-based microdisks on silicon substrates.

Zengcheng $\mathrm{Li}$ et al. investigated MOCVD growth conditions for AlGaN on (111) silicon substrate, a dissimilar substrate offering scalability and potential cost-effectiveness. The optimized conditions led to reduced carbon incorporation in n-AlGaN epitaxy as well as improved ultraviolet-A photoluminescence and electroluminescence properties. Liang Zhang et al. fabricated nanoporous template by electrochemical etching, and on top of which Al-rich n-AlGaN with improved crystalline quality was grown by MOCVD. Yahor V. Lebiadok et al. further simulated the mechanism of intermixing at the GaN/AlN heterointerfaces, and thus offering theoretical insights at the nanoscopic scale.

Linhao Guo et al. numerically designed and simulated aluminum nitride metalens across the ultraviolet-A-B-C regime. For improved deep-ultraviolet AlGaN-based LED design, Chunshuang Chu et al. numerically optimize the AlN mole fraction and position of the p-AlGaN insertion layer embedded in an electron blocking layer.

In the wide-bandgap nitride-semiconductor research, Nikhil Deep Gupta et al. implemented a photonic crystal light-trapping structure atop InGaN/GaN-superlattice for improved solar cells performance. The effect of weak magnetic field on $\mathrm{ZnO}$-film/Ag-nanoparticles structure was investigated by Charus M. Briskina et al., and the enhancement in exciton luminescence and random lasing was attributed to an increase in magnetoexciton recombination.

Finally, gratitude and acknowledgment are due to all authors, reviewers as well as the editorial and publication team of the Journal of Nanophotonics and the Special Section on Semiconductor UV Photonics.

Tien Khee Ng (Senior Member of IEEE, Member of SPIE, and Senior Member of OSA) received his $\mathrm{PhD}$ (2005) and MEng (2001) degrees in electrical and electronic engineering from Nanyang Technological University, Singapore. Currently, he is a senior research scientist with Boon S. Ooi's group at King Abdullah University of Science and Technology (KAUST), Saudi Arabia, and the co-principal investigator responsible for the Molecular Beam Epitaxy thrust of the KACST Technology Innovation Center for Solid-State Lighting at KAUST.

Jianchang Yan, $\mathrm{PhD}$, is a professor at the Institute of Semiconductors, Chinese Academy of Sciences. He received his bachelor's degree from Tsinghua University, and his $\mathrm{PhD}$ in microelectronics and solid-state electronics from the Graduate University of Chinese Academy of Sciences. In 2009, he joined the Semiconductor Lighting R\&D Center of CAS to continue the research work on III-nitride material and devices. He was visiting scholar of Paris Sud University from 2015 to 2016 . He is the leader of the III-nitride ultraviolet light-emitting device group, focusing on the research of $\mathrm{Al}(\mathrm{Ga}) \mathrm{N}$-based UV LEDs and UV laser diodes. 